dings aber zunächst nicht mehr als ein bloßer Anzeiger. Es ist ja heute ein eindeutiger SchluB aus Viskositätsänderungen auf das Wesen der durch diese verratenen Zustandsänderungen der Kolloide meist noch nicht ziehbar. So kann die Viskosität von Kolloiden zunehmen mit zunehmendem, aber auch mit abnehmendem Dispersitätsgrade. Gewiß haben nur jene Messungsangaben in der Biologie einen tiefen wissenschaftlichen Wert, „bei welchen zu übersehen ist, was für Aenderungen des Systems energetisch in der Viskosität zum Ausdruck kommen" [Schibig] ${ }^{29}$ ). Immer noch ist die

29) J. Schibig, loc. cit. auf der Versammlung der Faraday-Society im Jahre 1913 aufgeworfene Frage offen: „Was bedeuten, was meinen denn nur eigentlich alle diese Viskositätsmessungen, was zeigen sie eigentlich ?" Doch halten wir es vorerst lieber mit der tröstenden Bemerkung Wo. Ostwald's [S. 262] ${ }^{30}$ ): „Der phänomenologische Teil unserer Kenntnisse ist keineswegs weniger wichtig." Dem ist wohl ganz besonders dann so, wenn - wie bei der Viskositätsmessung lebenden Plasmas - der phänomenologische Teil der Hauptsache nach erst geschaffen werden muB.

so) Wo. Ostwald, Fußnote 9 .

\title{
Ueber das Zusammenwirken von Stäbchendoppelbrechung und Eigendoppelbrechung, ill.")
}

Von H. Ambronn (Jena).

(Eingegangen am 17 . Februar 1917.)

(Mitteilung aus dem Institut für Mikroskopie an der Universität Jena.)

VIII.

In der zweiten Mitteilung habe ich das optische Verhalten des bleibend deformierten Zelloidins eingehend geschildert. Im Verlauf der Gangunterschiede, wie er durch das Verändern des Brechungsexponenten der Imbibitionsflüssigkeit bei den "um etwa 100 Proz. verlängerten Streifen hervorgerufen wird, sind vor allem zwei Eigentümlichkeiten bemerkenswert. Bei der allmählichen Steigerung der Werte von 1,33 bis 1,72 tritt für alle Farben des sichtbaren Spektrums zweimal optische Isotropie und damit in Verbindung eine zweimalige Umkehr des Vorzeichens der Gesamtdoppelbrechung ein. Schon durch den sehr einfachen Versuch, der auf S. 274 und 275 in Mitteilung II beschrieben worden ist, kann man sich leicht von diesem merkwürdigen Verhalten des Zelloidins überzeugen; je nach der Höhe des Brechungsexponenten der eingelagerten Flüssigkeit ist die sog. akzidentelle Doppelbrechung der gedehnten Streifen positiv, Null oder negativ. In dieser Beziehung weicht also das Zelloidin im imbibierten Zustande von allen anderen bisher auf ihre akzidentelle Doppelbrechung untersuchten Körper ganz wesentlich ab. Man kannte zwar auch bisher schon Körper, bei denen die akzidentelle Doppelbrechung im Verlauf einer länger andauernden Dehnung ihr Vorzeichen (1916)

1) Vgl. Koll.-Zeitschr. 18, 90-97 und 273-281 einmal umkehrt, so z. B. das Kirschgummi ${ }^{2}$ ), die Guttapercha ${ }^{3}$ ), das mit echtem Kampfer hergestellte Zelluloid ${ }^{4}$ ), aber diese Umkehr erfolgt stets nur bei einem bestimmten Deformationsgrad und ist gänzlich unabhängig von einer Aenderung des Brechungsexponenten des Körpers oder eines seiner Bestandteile.

Die in Fig. 3 in Mitteilung II dargestellten Kurven lassen deutlich erkennen, in welcher Weise die Höhe des Gangunterschieds von der Differenz der Brechungsexponenten der Grundsubstanz und der eingelagerten Flüssigkeit abhängt. Erreicht diese Differenz den Wert Null, so zeigt auch die Kurve des Gangunterschieds ein Minimum. Dieses Minimum tritt natürlich nicht bei allen Wellenlängen zugleich auf, sondern seine Lage ist abhängig vom Gange der Dispersion, da jene Differenz der Brechungsexponenten nicht für alle Wellenlängen zugleich verschwinden kann. Die beträchtlichen Schwankungen in der Höhe der Gangunterschiede und die noch stärkere Verschiedenheit in der Dispersion der Doppelbrechung sind dabei be-

2) Ueber das optische Verhalten und die Struktur des Kirschgummis (Ber. d. Deutsch. Bot. Ges. 7, 103 bis 114, 1889).

3) Ueber Anomalien bei der akzidentellen Doppelbrechung (Ber. d. Kgl. Sächs. Ges. d. Wiss. math.-phys. K1. Naturw. Teil 50,1-31, 1898).

4) Ueber anomale Doppelbrechung beim Zelluloid (Ber. d. Kgl. Sächs. Ges. d. Wiss. math.- physik. Kl. Naturw. Teil 63, 249-257 und 402-406, 1911); vgl. auch Koll.-Zeitschr. 9, $147-153$ (1911). 
sonders bemerkenswert. Wie bereits in der ersten Mitteilung gezeigt werden konnte, finden sich dieselben Eigenschaften bei der $S t a ̈ b$ chendoppelbrechung im Sinne O. Wiener's (vgl. Mitteilung I). Diese ist aber, wenigstens soweit es sich um farblose Körper handelt, stets positiv; denn ein System parallel gestellter Stäbchen, die von einem Medium umgeben sind, dessen Brechungsexponent von dem der Stächensubstanz abweicht, verhält sich nach den Wiener'schen Untersuchungen wie ein einachsiger, positiver Kristall, dessen optische Achse den Längsachsen der Stäbchen parallel steht. Die Beobachtungen am Zelloidin haben nun zwar mit Sicherheit ergeben, daß die Stärke der Doppelbrechung mit dem Werte von $\mathrm{n}_{2}$ (Brechungsexponent der Imbibitionsflüssigkeit) veränderlich ist, zugleich aber auch, $\mathrm{daB}$ die Doppelbrechung nur innerhalb zweier ganz bestimmten Bezirke der Werte von $\mathrm{n}_{2}$ positiv ist, während sie bei anderen Werten negativ wird oder sogar völlig verschwindet. Es kann deshalb die beobachtete Anisotropie nicht allein auf die Stäbchendoppelbrechung zurückgeführt werden, es mub noch eine andere Komponente hinzukommen, die das entgegengesetzte Vorzeichen besitzt. Nur auf diese Weise kommt man zu einer befriedigenden Erklärung des so eigentümlichen Verlaufs der Gangunterschiede. Diese zweite Komponente $\mathrm{muB}$ aber rein zur Geltung kommen, wenn $\mathrm{n}_{2}$ gleich $n_{1}$ (Brechungsexponent des Zelloidins) wird, denn dann verschwindet nach der Theorie die Stäbchendoppelbrechung vollständig und die noch übrig bleibende Anisotropie muß also mit einer Eigenschaft der Stäbchen selbst zusammenhängen. Ich habe diese Komponente deshalb als Eigendoppelbrechung bezeichnet; sie wird während des ganzen Verlaufs der Beobachtungen sowohl in ihrer Stärke, wie in ihrem Charakter unverändert bleiben, wenn dic Deformation dieselbe bleibt und die Stäbchensubstanz durch die imbibierenden Flüssigkeiten weder chemische noch physikalische Veränderungen erfährt. Wir hätten es also mit dem Zusammenwirken zweier Komponenten zu tun, von denen die eine konstant, die andere aber mit dem Werte von $n_{2}$ veränderlich ist. Beide Komponenten haben jedoch entgegengesetztes Vorzeichen, demnach muß auch die Resultierende in zwei ganz bestimmten Fällen Null werden, außerdem muB eine zweimalige Umkehr des Vorzeichens eintreten. Hat dagegen auch die Eigendoppelbrechung den positiven Charakter, so wird zwar der Verlauf der Kurven der Gangunterschiede im wesentlichen ähnlich sein, die Umkehr des Vorzeichens jedoch muB dann ebenso unterbleiben wie das Eintreten der Isotropie. Wie schon früher mehrfach erwähnt wurde ${ }^{5}$ ), zeigt die durch Denitrierung aus dem Zelloidin gewonnene Zellulose ein solches Verhalten. Da aber auch in diesem Falle die Stäbchendoppelbrechung mitwirkt, so muß sich ebenfalls eine deutlich bemerkbare Veränderung in der Dispersion der Gesamtdoppelbrechung ergeben, wenn sie auch nicht eine so starke Abweichung in den Interferenzfarben hervorrufen kann. Die in dem nächsten Abschnitt zusammengestellten Beobachtungen werden zeigen, dab das optische Verhalten der Zellulose in der Tat dem eben Gesagten entspricht und daB nur untergeordnete Abweichungen auftreten, die auf andere Weise ihre Er= klärung finden können.

IX.

Die Vorbereitung der zu den Beobachtungen benutzten Objekte geschah stets in der Weise, daß die um 100 Proz. ihrer ursprünglichen Länge bleibend deformierten Zelloidinstreifen etwa acht Tage hindurch unter mehrmaliger Erneuerung der Flüssigkeit in Ammoniumsulfid verblieben und danach ebenfalls mehrere Tage in destilliertem Wasser genügend ausgewaschen wurden. $\mathrm{DaB}$ auf diese. Weise eine vollständige Denitrierung erzielt wird, davon kann man sich leicht auf zweifache Art überzeugen. Zunächst läßt sich feststellen, daß Schnitte durch solche Streifen in ihrer ganzen Ausdehnung die Zellulosereaktion mit Chlorzinkjod ergeben und dabei denselben sehr starken Dichroismus zeigen, den gewöhnliche Zellulosemembranen mit diesem Reagens erhalten. Ferner kann man sich durch längere Behandlung der denitrierten Streifen mit einem Alkohol-Aethergemisch überzeugen, daß nichts mehr in Lösung geht, daB also alles Zelloidin wirklich in Zellulose umgewandelt ist. Durch die Denitrierung wird, wie ich früher schon erwähnt habe, eine geringe Formveränderung verursacht; aber diese findet nicht etwa in der Weise statt, dab die durch Dehnung hervorgerufene Verlängerung in erster Linie verkürzt wird, sondern es ergibt sich eine fast gleichmäßige Veränderung in allen Richtungen. Ein Beispiel möge dies erläutern: Ein Streifen, der vor der Denitrierung $20 \mathrm{~mm}$ lang, $1,8 \mathrm{~mm}$ breit und $1,25 \mathrm{~mm}$ dick war,

5) Zeitschr. f. wiss. Mikroskopie 32, 5i (1915); ferner Koll.-Zeitschr. 18, 280 (1916). 
zeigte nach vollständiger Denitrierung die Maße : $18.2 \mathrm{~mm}, 1,6 \mathrm{~mm}$ und $1,1 \mathrm{~mm}$, er hatte also in jeder Richtung eine Verkürzung um etwa 9-10 Proz. erfahren, dabei aber seine prismatische Gestalt vollständig beibehalten. Es ist nun beachtenswert, daß die Formveränderung eines Streifens von denselben Dimensionen aus frischem, nicht deformiertem Zelloidin bei derselben Behandlung fast genau dieselbe war. Obwohl bei dem ganzen Vorgang der Denitrierung eine sehr weitgehende chemische Veränderung der Zelloidinteilchen herbeigeführt wird, so ist doch die damit verbundene Formveränderung eine recht geringe. Dies ist jedoch durchaus nicht so überraschend, wie es auf den ersten Blick scheint, denn man weiß, daB auch bei dem umgekehrten Vorgang, nämlich bei der Nitrierung der Zellulosefasern ${ }^{6}$ ), die äuBere Form fast vollständig beibehalten wird. Ganz anders verhält es sich aber mit der Stärke der Doppelbrechung, die vor und nach der Denitrierung festzustellen ist. Wie aus der Vergleichung der Tabelle $\mathrm{V}$ in Mitt. II (Koll.-Zeitschr. 18, 270) und der folgenden Tabelle VII sofort zu ersehen ist, wird die Doppelbrechung nach der Umwandlung um mehr als das Doppelte erhöht, denn die Zahlen für die Gangunterschiede in der Tabelle $\mathrm{V}$ gelten für einen $3,7 \mathrm{~mm}$ dicken Zelloidinstreifen, die in der Tabelle VII dagegen für einen nur $1 \mathrm{~mm}$ dicken Zellulosestreifen. Diese sehr starke Veränderung im optischen Verhalten ist ebenfalls nicht überraschend, wenn man bedenkt, daß bei der Nitrierung der Zellulosefasern ein ganz ähnlicher Vorgang sich abspielt. Es möge genügen, auf diese großen Verschiedenheiten im Verhalten der reinen Zellulose und der Nitrozellulose hier nur kurz ninzuweisen; ich werde später eingehender darauf zurückkommen.

Als Imbibitionsflüssigkeiten für die Zellulosestreifen dienten: Wasser, Aethylalkohol, Benzylalkohol und Monobromnaphthalin. Man kann jede dieser Flüssigkeiten durch die im Brechungsexponenten nächst höhere allmählich verdrängen und erhält somit Mischungen, deren Exponenten zwischen 1,33 und 1,66 liegen. Beim Zelloidin konnte ich im wesentlichen mit Lösungen von Kaliumquecksilberjodid in verschiedenen Verdünnungen auskommen, wodurch die Beobachtungen sehr vereinfacht wurden. Leider ließ sich dieses Salz bei der Zellulose

6) Hans Ambronn, Ueber die Aenderung des optischen Verhaltens der Zellulose bei der Nitrierung (Diss., Jena 1914). Vgl. auch Koll.-Zeitschr. 13, 200 bis 207 (1913). nicht verwenden, đa seine Lösungen eine beträchtliche Quellung verursachten. Nach mehreren Versuchen mit anderen Flüssigkeiten wählte ich die vier bereits genannten, weil die Streifen in ihnen keinerlei bemerkbare Formveränderungen zeigten. Wenn auch der Spielraum der Brechungsexponenten etwas kleiner war, als bei den Versuchen am Zelloidin, so genügte er doch völlig, un einen Ueberblick über den Verlauf der Gesamtdoppelbrechung und damit auch der dabei wirksamen Stäbchendoppelbrechung zu erhalten.

Die Messungen der Gangunterschiede wurden wie früher beim Zelloidin an zahlreichen Streifen von verschiedener Dicke sowohl in aufsteigender wie in absteigender Reihenfolge durchgeführt. Die Mittelwerte aus den gewonnenen Zahlen wurden dann auf eine Streifendicke von $1 \mathrm{~mm}$ umgerechnct. Neben dem Kompensatorokular nach $H$. Siedento $f$ benutzte ich diesmal auch noch zur bequemeren Orientierung eine Anzahl Glimmerplättchen von allmählich steigenden Gangunterschieden, die für die in Betracht kommenden Wellenlängen genau geeicht waren. Als Lichtquellen dienten wieder die Quecksilberbogenlampe mit den $\mathrm{K}$ ö hle r'schen Lichtfiltern für die Wellenlängen $436 \mu \mu$ und $546 \mu \mu$ und eine MikroskopierNernstlampe nach $H$. Siedent op $f$ in Verbindung mit zwei Farbglasplatten; davon war die eine die dunkle Rotglasplatte, die ich früher schon benutzte, und die andere ein Blauglas. Bei den hohen Gangunterschieden, wie sie die dickeren Streifen zeigten, erwies sich die Rotglasplatte als nicht genügend, da sie ein $\mathrm{zu}$ breites Gebiet aus dem Rot durchlieh, während beim Zufügen des Blauglases nur eine schmale Zone zwischen $670 \mu \mu$ und $680 \mu \mu$ wirksam wurde, bei der auch recht hohe Gangunterschiede noch genügend schwarze Interferenzstreifen ergaben, Im folgenden ist dieses Gebiet stets mit $675 \mu \mu$ bezeichnet. Nach den für das Verhalten des Zelloidins bereits gegebenen Darlegungen sind die Zahlen in Tabelle VII und die Kurven in Fig. 5 ohne weiteres verständlich. Die Ordinaten bedeuten die beobachteten Gangunterschiede $\gamma_{0}$ für e in en Streifen von $1 \mathrm{~mm}$ Dicke, und die Abszissen sind die Brechungsexponenten der Imbibitionsflüssigkeiten für die $\mathrm{D}$-Linie.

Es ist also auch bei der Zellulose eine beträchtliche Veränderung der Gesamtdoppelbrechung mit der Steigerung des Brechungsexponenten der Imbibition sflüssigkeit verknüpft ; die Gangunterschiede gehen aber hier für keine 
Tabelle VII

\begin{tabular}{c||c|c|c||c}
\hline \hline$\left(\mathbf{n}_{\mathbf{2}}\right)_{\mathrm{D}}$ & $436 \mu \mu$ & $\begin{array}{c}\gamma_{0} \\
546 \mu \mu\end{array}$ & $675 \mu \mu$ & $\nu_{0}$ \\
\hline & 436 & \\
\hline 1,33 & 10,95 & $\mathbf{8 , 5 0}$ & $\mathbf{6 , 5 5}$ & 1,08 \\
1,35 & 10,70 & $\mathbf{8 , 3 5}$ & $\mathbf{6 , 4 5}$ & 1,08 \\
1,37 & 10,48 & 8,24 & 6,33 & 1,07 \\
1,39 & 10,03 & 7,85 & 6,02 & 1,08 \\
1,41 & 9,44 & 7,38 & 5,70 & 1,07 \\
1,43 & 8,90 & 6,94 & 5,35 & 1,08 \\
1,45 & 8,48 & 6,59 & 5,08 & 1,08 \\
1,47 & 8,14 & 6,30 & 4,85 & 1,09 \\
1,49 & 7,87 & 6,10 & 4,68 & 1,09 \\
1,51 & 7,77 & 6,02 & 4,60 & 1,09 \\
1,53 & 7,72 & 5,96 & 4,55 & 1,10 \\
1,55 & 7,75 & 5,99 & 4,53 & 1,10 \\
1,57 & 7,86 & 6,04 & 4,55 & 1,11 \\
1,59 & 8,07 & 6,17 & 4,65 & 1,12 \\
1,61 & 8,46 & 6,32 & 4,81 & 1,13 \\
1,63 & 8,83 & 6,65 & 4,98 & 1,14 \\
1,65 & 9,47 & 7,14 & 5,28 & 1,16 \\
1,661 & 9,95 & 7,47 & 5,48 & 1,17 \\
\hline
\end{tabular}

In der letzten Reihe der Tabelle sind die Zahlen für die Dispersion der Gesamtdoppelbrechung wie früher in der Form

wiedergegeben.

$$
\nu_{0}=\frac{\left(n_{a}-n_{0}\right)_{436} \mu \mu}{\left(n_{a}-n_{0}\right)_{675} \mu \mu}
$$

Der Brechungsexponent 1,661 ist der des von mir benutzten Monobromnaphthalins bei Zimmertemperatur.

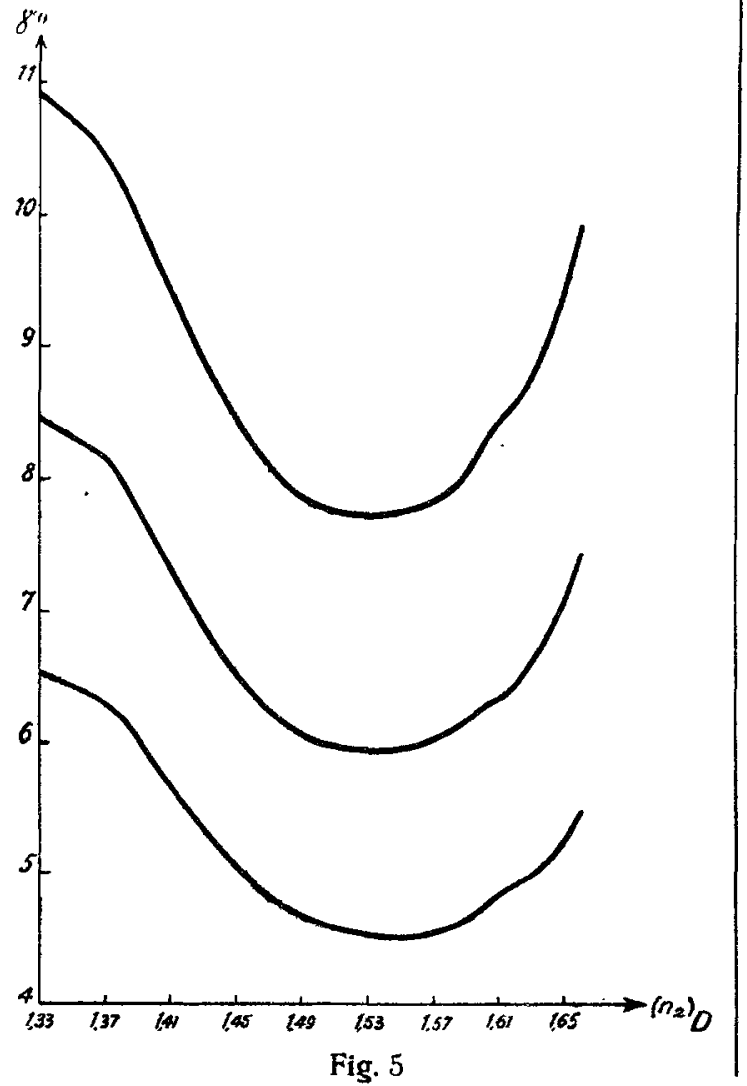

Wellenlänge auf Null herab, sie behalten stets dasselbe Vorzeichen. Dort, wo die Kurven die tiefsten Stellen erreichen, wird, wie auch beim Zelloidin, die Eigendoppelbrechung der Zellulose allein zur Geltung kommen. Würde man die Werte von $\left(n_{2}\right)_{D}$, die jenen Stellen entsprechen, mit hinreichender Genauigkeit bestimmen können, so könnte man daraus auch, unter Berücksichtigung der Dispersion der angewandten Flüssigkeiten, die Dispersion der Zellulose berechnen. Wie schon früher hervorgehoben wurde, ist aber die Feststellung der Lage der Minima, wegen des an diesen Stellen sehr flachen Verlaufes der Kurven, mit Schwierigkeiten verknüpft. Es handelte sich ja hier auch gar nicht darum, die Dispersion der Zellulose zu ermitteln, aber es hatte doch ein gewisses Interesse, zu prüfen, ob der sich ergebende Wert mit einem größeren Fehler behaftet sei. Bei der durchgeführten Näherungsrechnung, auf die ich hier nicht weiter eingehen will, ergab sich für das in Betracht kommende Gebiet zwischen $436 \mu \mu$ und $675 \mu \mu$ der Wert von etwa 0,02 , der für einen festen Körper vom mittleren Brechungsexponenten 1,54, durchaus wahrscheinlich ist. Eine ähnliche Unsicherheit besteht, wie gleichfalls früher schon ausgeführt wurde, bei der Berechnung der Eigendoppelbrechung aus den Minimalwerten für die Gangunterschiede: $7,72,5,95,4,53$; dazu kommt noch, daß der Betrag für $\delta_{1}$, d. h. der Anteil an der Gesamtdicke des Streifens ebenso, wie beim Zelloidin, nur mit einiger Wahrscheinlichkeit zu bestimmen ist. Wählt man unter Berücksichtigung der Angaben auf S. 279 in Mitteilung II $\delta_{1}=0,25$, so erhält man für die Eigendoppelbrechung $\alpha_{1}$ folgende Werte:

$$
\left(\alpha_{1}\right)_{436} \mu \mu=0,01346,\left(\alpha_{1}\right)_{546 \mu \mu}=0,01301 \text {, }
$$
$\left(\alpha_{1}\right)_{675} \mu \mu=0,01223$, und daraus $\nu_{1}=1,10$.

Für die Berechnung der Werte $\alpha_{2}$, der Stärke der Stäbchendoppelbrechung, fällt dagegen diese Unsicherheit weg, da hierbei ja die Gesamtdicke des Streifens, für den in Tabelle VIl dargestellten Fall also $1 \mathrm{~mm}$, in Betracht kommt; man hat nur die Differenzen $\gamma_{0}-\gamma_{1}$ zu bilden und daraus für die verschiedenen Wellenlängen die zugehörige Stärke der Stäbchendoppelbrechung $\alpha_{2}$ zu berechnen. In der Tabelle VIII sind die gefundenen Zahlen zusammengestellt und auberdem in der letzten Reihe die Werte für $\nu_{2}$ angegeben, wie sie sich direkt aus den Zahlen für $\gamma_{2}=\gamma_{0}-\gamma_{1}$ ergeben. Ferner ist in Fig. 6 wieder wie früher der Verlauf der Werte $\alpha_{\text {g. }}$ für die Wellenlänge von $546 \mu \mu$ dar- 
gestellt. Man sieht daraus, dab dasselbe, was auf S. 279 zur Fig. 4 gesagt worden ist, auch hier im wesentlichen seine Giltigkeit behält.

Tabolle VIII.

\begin{tabular}{c||c|c|c||c}
\hline \hline$\left(\mathrm{n}_{2}\right)_{\mathrm{D}}$ & $436 \mu \mu$ & $\begin{array}{c}\alpha_{2} 10^{-3} \\
546 \mu \mu\end{array}$ & $675 \mu \mu$ & $\nu_{2}$ \\
\hline \hline 1,33 & 1,40 & 1,39 & 1,36 & 1,03 \\
1,35 & 1,31 & 1,31 & 1,30 & 1,00 \\
1,37 & 1,20 & 1,25 & 1,22 & 0,99 \\
1,39 & 1,01 & 1,04 & 1,00 & 1,00 \\
1,41 & 0,75 & 0,80 & 0,79 & 0,95 \\
1,43 & 0,51 & 0,54 & 0,55 & 0,92 \\
1,45 & 0,33 & 0,35 & 0,37 & 0,90 \\
1,47 & 0,18 & 0,19 & 0,22 & $0,85)$ \\
1,49 & 0,06 & 0,08 & 0,10 & $0,66 \mathrm{~L}$ \\
1,51 & 0,02 & 0,034 & 0,047 & $0,48 \mathrm{~A}$ \\
1,53 & 0 & 0,003 & 0,014 & 0 \\
1,55 & 0,013 & 0,02 & 0 & $\infty$ \\
1,57 & 0,06 & 0,05 & 0,014 & 4,5 \\
1,59 & 0,15 & 0,12 & 0,08 & 1,9 \\
1,61 & 0,32 & 0,25 & 0,19 & 1,7 \\
1,63 & 0,48 & 0,40 & 0,30 & 1,6 \\
1,65 & 0,76 & 0,65 & 0,51 & 1,6 \\
1,661 & 0,97 & 0,83 & 0,64 & 1,5 \\
& & &
\end{tabular}

*) Die Buchstaben B, L, A und $\mathrm{Ch}$ bedeuten hier wieder dasselbe wie in Tabelle $\mathrm{V}$.

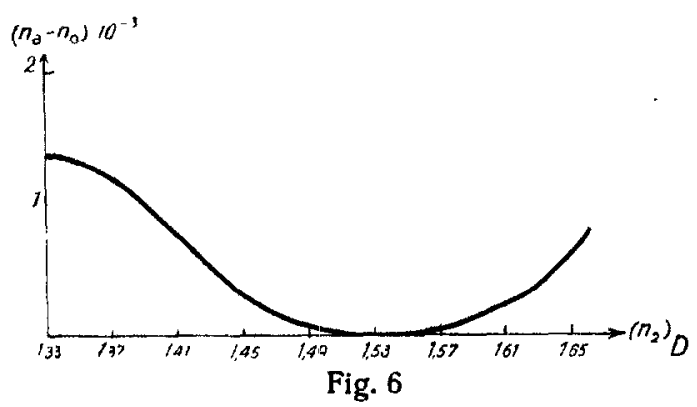

Vergleicht man die Tabellen $V$ und VII sowie die zugehörigen Kurventafeln Fig. 3 und Fig. 5 genauer mit einander, so erkennt man sofort, daB das Verhalten der Zellulose mit dem des Zelloidins in einem wichtigen Punkte fast ganz übereinstimmt. In beiden Fällen sinkt zunächst die Kurve der Gangunterschiede der Gesamtdoppelbrechung mit wachsenden Werten von $\mathrm{n}_{2}$, bei den Werten zwischen 1,52 und 1,56 wird für die verschiedenen Wellenlängen nacheinander ein Minimum erreicht, und von da ab steigen die Kurven wieder mit weiter wachsendem $n_{2}$. Ganz dasselbe zeigt sich natürlich auch bei den Werten für die Stärke der Stäbchendoppelbrechung, die aus den beobachteten Gangunierschieden und der Streifendicke berechnet wurden. Zugleich fällt aber auch bei dem Betrachten der Kurven auf, daß bei der Zellulose der Verlauf der Kurven nicht so gleichmäBig ist wie beim Zelloidin. Es macht sich hier sowohl beim Sinken der Werte, wie auch beim Ansteigen eine Störung bemerkbar, die auf dem absteigenden Ast stärker als auf dem ansteigenden hervortritt. Ich vermutete zunächst irgend einen versteckten Beobachtungsfehler, doch ergab sich bei den sehr zahlreichen Versuchsreihen, die den angeführten Zahlen zu Grunde liegen, sowohl in aufsteigender wie in absteigender Reihenfolge fast immer dasselbe Resultat. Die Versuche wurden stets in der Weise angestellt, daß die Streifen zuerst in reinem Wasser, darauf in verschiedenen Gemischen von Aethylalkohol und Wasser, dann in absolutem Alkohol und von $\mathrm{da} a b$ in Gemischen von Alkohol und Benzylalkohol bis zum reinen Benzylalkohol und schließlich in Gemischen von Benzylalkohol und Monobromnaphthalin bis zum reinen Monobromnaphthalin untersucht wurden. In jeder dieser Flüssigkeiten blieben die Streifen mindestens 24 Stunden, oft auch noch länger, ehe die Messung der Gangunterschiede vorgenommen wurde. Man darf somit wohl annehmen, daB innerhalb dieser Zeit jeweils eine vollständige Durchdringung der verhältnismäfig kleinen Objekte - es handelte sich doch stets nur um kurze Streifen von 3-4 mm Länge und 1-1,5 mm Dicke - stattgefunden hatte und das Brechungsvermögen der umgebenden Flüssigkeit auch zur Wirkung kam. Das ist aber doch an zwei ganz deutlich sich abhebenden Stellen offenbar nicht der Fall gewesen; denn sonst müßten diese Störungen wegfallen, zumal sie auch beim Zelloidin gar nicht zu beobachten waren. Worauf nun diese Ungleichmäßigkeiten, deren Betrag ja durchaus nicht beträchtlich ist, beruhen, vermag ich zur Zeit nicht mit Sicherheit anzugeben. Meine Vermutung geht dahin, daB das Adsorptionsvermögen der Zelluloseteilchen sich gegenüber den vier Flüssigkeiten verschieden verhält, was wohl auch von vornherein durchaus nicht unwahrscheinlich ist. Bestehen aber solche Verschiedenheiten, so wäre es auch ohne weiteres verständlich, wenn z. B. beim Uebergang vom Wasser zum Benzylalkohol durch den absoluten Alkohol hindurch und ebenso beim Verdrängen des Benzylalkohols durch das Monobromnaphthalin zeitweise geringe Unterschiede im Brechungsvermögen der Imbibitionsflüssigkeit und der umgebenden Mischung, deren Brechungsexponenten man direkt bestimmt, erhalten bleiben. $\mathrm{DaB}$ das Adsorptionsvermögen der Zellulose gegen Wasser 
stärker als gegen Alkohol ist, hätte gar nichts Ueberraschendes; und ein ganz ähnliches Verhältnis könnte zwischen dem Benzylalkohol und dem Monobromnaphthalin bestehen. Wird z. B. das Wasser den Zellulosestäbchen durch den absoluten Alkohol nicht vollständig entzogen und erst durch den hinzukommenden Benzylalkohol völlig verdrängt, so müßte sich etwas Aehnliches im Verlauf der Kurven zeigen, wie wir es tatsächlich beobachten. Und ganz entsprechend würden die Dinge liegen, wenn die Zellulose gegenüber dem Benzylalkohol ein stärkeres Adsorptionsvermögen als gegen das Monobromnaphthalin besäße. Ich möchte jedoch ausdrücklich betonen, da $B$ diese Bemerkungen nicht mehr als eine Vermutung enthalten sollen; denn zur Entscheidung dieser Frage bedürfte es weiterer systematisch durchgeführter Untersuchungen. Daß bei dem Verhalten des Zelloidins derartige Störungen nicht beobachtet wurden, ließe sich dann auf Grund jener Vermutung wohl einfach damit erklären, daß hier eigentlich nur eine Flüssigkeit, nämlich das Kaliumquecksilberjodid in wässeriger Lösung in sehr verschiedenen Konzentrationen in Betracht kam. Hier dürften deshalb kaum größere Verschiedenheiten im Adsorptionsvermögen, die solche Störungen hervorrufen würden, zur Geltung kommen.

Abgesehen von den beiden Ungleichmäßig. keiten im Verlauf der Gangunterschiede, die übrigens, wie aus der Tabelle und der Kurventafel hervorgeht, für alle untersuchten Wellenlängen an denselben Stellen liegen, nämlich in der Nähe der Werte 1,37 und 1,61 für $n_{2}$, zeigt das Verhalten der Zellulose und des Zelloidins in dem wichtigsten Punkte gute Uebereinstimmung. Die Gesamtdoppelbrechung ist in beiden Fällen von dem Brechungsexponenten der eingelagerten Flüssigkeit abhängig und zwar in derselben Weise, wie dies nach der Theorie der Stäbchendoppelbrechung zu erwarten war. Berechnet man nach Berücksichtigung der Eigendoppelbrechung den Gang der Stärke der Stäbchendoppelbrechung, so ergibt sich auch für die Zellulose der charakteristische Verlauf in der Dispersion der Stäbchendoppelbrechung, wie aus den $\nu_{2}$-Werten in der Tabelle VIII deutlich hervorgeht. Auch das allmähliche Ansteigen der $y_{0}$-Werte in Tabelle VIl stimmt damit gut überein, denn hier fällt natürlich eine zweimalige Umkehr im Vorzeichen der Doppelbrechung weg, da ja beide Komponenten, aus denen sich die Gesamtdoppelbrechung zusammensetzt, dasselbe Vorzeichen besitzen. kann sich deshalb, wie schon erwähnt wurde, keine so starke Abweichung ia der Reihenfolge der Interferenzfarben zeigen, wie sie für das Zelloidin charakteristisch ist. Ganz besonders bemerkenswert ist es, daß die Zahlen für die Stärke der reinen Stäbchendoppelbrechung in den beiden Fällen so gut übereinstimmen, während doch die Eigendoppelbrechung, wie sie sich aus den Minima der Kurven für die Gangunterschiede ergibt, so beträchtliche Unterschiede nicht bloß in ihrer Stärke, sondern auch in ihrem Vorzeichen aufweist. Allerdings ist durch die Denitrierung eine ganz wesentliche chemische und auch physikalische Veränderung eingetreten, aber man darf nach früheren Untersuchungen annehmen, daB der Aufbau der Systeme aus Stäbchen, die von Flüssigkeit umhüllt sind, im wesentlichen derselbe geblieben ist ; auch die Beibehaltung der Form nach einem so starken Eingriff spricht entschieden für die Berechtigung dieser Annahme. Denn so, wie nicht nur bei der Nitrierung der Zellulosefasern, sondern auch bei der nachfolgenden Denitrierung die Faserform vollständig erhalten bleibt, die Anordnung der die Fasern aufbauenden Teilchen also unverändert fortbesteht, so darf man auch hier annehmen, daß die Anordnung der stäbchenförmigen Teilchen in den Zelloidinund in den Zellulosestreifen im wesentlichen dieseibe bleibt. Ist dies aber in der Tat so, dann muß auch die Stärke der Stäbchendoppelbrechung in diesen beiden Fällen nahezu gleich groß sein; denn die Werte für $n_{1}$, die Brechungsexponenten der Stäbchen, sind bei dem Zelloidin und der Zellulose offenbar nur wenig von einander verschieden, und damit müssen die Differenzen $n_{1}-n_{2}$, die für die Stärke der Stäbchendoppelbrechung maßgebend sind, ebenfalls nur geringe Unterschiede zeigen. Ich werde später auf diesen wichtigen Punkt nochmals zurückkommen.

Am Schlusse dieses Abschnittes möge noch kurz auf die schon erwähnten getingen Dimensionsänderungen der Streifen bei der Denitrierung hingewiesen werden. Es wurde auch schon angedeutet, daß dieselben Dimensionsänderungen eintreten, wenn man nicht gedehnte, sondern frische optisch vollständig isotrope Streifen denitriert. Solche Streifen zeigen nun nach der Umwandlung, auch ohne dab sie irgend eine künstliche Spannung erfahren haben, eine zwar schwache aber doch immerhin deutliche Doppelbrechung; die längere Achse des Indexellipsoids liegt auch hier parallel zur Längsrichtung der Streifen. Diese Doppel- 
brechung hat also, wenigstens bei jener prismatischen Form der Streifen, denselben Charakter, wie die durch starke Dehnung hervorgerufene; sie zeigrt aber auch dieselbe Abhängigkeit von dem Brechungsexponenten der eingelagerten Flüssigkeit. Bringt man derartige Streifen nacheinander in Wasser, Aethylalkohol, Benzylalkohol und Monobromnaphthalin, so ist die Doppelbrechung im Benzylalkohol am schwächsten und bei dünnen Streifen kaum bemerkbar, in Wasser erreicht sie den höchsten Betrag, in Monobromnaphthalin ist sie etwas geringer. Dazwischen treten natürlich alle Uebergänge auf. Das ist also ganz dasselbe Verhalten, das auch die vorher stark gedehnten Streifen aufweisen, und man darf daher wohl mit Recht annehmen, daß hier ebenfalls schon ein Zusammenwirken von Stäbchen- und Eigendoppelbrechung vorliegt. Hält man an der Annahme fest, dab die optische Isotropie des angedehnten Zelloidins auf die regellose Lagerung der stäbchenförmigen Teilchen zurückzuführen sei, so ist es auch verständlich, wenn allein durch die Dimensionsänderungen bei der Denitrierung zu Zellulose eine gewisse Umlagerung dieser Stäbchen eintritt, und die Summe der Einzelwirkungen jetzt von Null verschieden ist. Liegt, wie in unserem Falle, ein prismatischer Streifen vor, in dem die Dimension in einer Richtung die in den andern bedeutend überwiegt, so wird die bei der Denitrierung eintretende Querkontraktion eine optische Anisotropie hervorrufen, vorausgesetzt, daB die Stäbchen an sich schon optisch anisotrop sind. Es wirkt eben in diesem Falle die Querkontraktion so, als ob der Streifen durch die Denitrierung einen allseitigen Druck senkrecht zur Längsrichtung und dadurch eine bleibende Deformation erfahren hätte. Bedenkt man außerdem noch, dab, wie ebenfalls schon früher hervorgehoben wurde, bei der Denitrierung eine Vermehrung der Trockensubstanz, also eine Verringerung der Imbibitionsflüssigkeit eintritt, und damit dieselbe Anzahl der Stäbchen in einem in der Querrichtung engeren Raum Platz finden muß, so hat die entstehende Anisotropie gar nichts Ueberraschendes. Eine genauere quantitative Untersuchung hierüber würde sehr erwünscht sein; da aber die eben geschilderten Veränderungen für die zunächst zu erörternden Beziehungen żwischen Stäbchen- und Eigendoppelbrechung nur von nebensächlicher Bedeutung sind, so wurden jene Fragen nicht weiter verfolgt, als es für den Hauptzweck nötig erschien. Der Verlauf der Gangunter- schiede, wie er oben ausführlich geschildert wurde, wird nämlich dadurch nicht weiter beeinfluBt, dab durch die Denitrierung an sich schon eine schwache Eigendoppelbrechung entsteht; diese addiert sich eben zu der weit beträchtlicheren, die durch die starke bleibende Deformation hervorgerufen ist. Es wird also an dem Gesamtergebnis, soweit es die Feststellung der Stäbchendoppelbrechung und ihres Zusammenwirkens mit der Eigendoppelbrechung betrifft, gar nichts geändert. Nur wenn man den Betrag der Eigendoppelbrechung für eine bestimmte bleibende Verlängerung genau berechnen will, muB man die durch die Denitrierung allein schon entstandene berücksichtigen. $\mathrm{Da}$ aber die genaue quantitative Bestimmung der Stärke der Eigendoppelbrechung aus verschiedenen früher schon angedeuteten Gründen auf Schwierigkeiten stöBt, so hat es für unsere Fragen keinen $Z$ weck, neben dem weit überwiegenden EinfluB der wirklich vollzogenen Spannungsdeformation jenen geringen durch die Denitrierung allein eingetretenen durch die Rechnung scharf hervorzuheben. Dagegen hat es von anderen Gesichtspunkten aus ein gewisses Interesse, $z \mathfrak{u}$ untersuchen, in welcher Weise die ursprüngliche Form der Zelloidinstücke auf die Art der Doppelbrechung nach der Denitrierung einen Einfluß hat. Es ist von vornherein $z u$ erwarten, daß ein solcher Einfluß besteht, daß z. B. ein Würfel oder eine kreisförmige Scheibe sich anders verhalten, als ein prismatischer Streifen, dessen Länge den Querdurchmesser bedeutend übertrifft. $\mathrm{DaB}$ in dieser Beziehung ein ganz charakteristischer Unterschied besteht, haben die zur vorläufigen Orientierung angesteliten Versuche schon mit Sicherheit ergeben und ich hoffe, in einer späteren Mitteilung darüber genauer berichten zu können.

\section{$\mathrm{X}$.}

Das besonders merkwürdige Verhalten des Zelloidins hatte die Veranlassung zu der hier vertretenen Annahme gegeben, dab die akzidentelle Doppelbrechung dieses Körpers im imbibierten Zustand auf das Zusammenwirken zweier Komponenten von entgegengesetztem Vorzeichen zurückzuführen sei. Auf Grund dieser Annahme lieb sich auch sofort der überraschende zweimalige Durchgang durch den isotropen Zustand erklären. Allerdings konnte dabei die Stärke der Doppelbrechung in beiden Komponenten nicht zugleich konstant sein, wie dies bei den Apophylliten oder bei den ganz 
áhnlich sich verhaltenden Mischkristallen aus dem optisch positiv einachsigen Bleidithionat und dem isomorphen aber negativen Strontiumdithionat der Fall ist ${ }^{7}$ ). Denn hierbei kann nur ein einmaliger Durchgang durch den isotropen Zustand bei einer ganz bestimmten $\mathrm{Zu}$ sammensetzung der Mischung auftreten, wie es auch die Erfahrung bestätigt hatte. Soll dagegen eine zweimalige Nullwirkung stattfinden, so mub die eine Komponente eine Doppelbrechung hervorrufen, deren Vorzeichen zwar stets dasselbe bleibt, deren Stärke aber in einer ganz bestimmten Weise veränderlich ist. Als eine solche Art von Doppelbrechung konnte nun in dem Zwei - Stoff - System des imbibierten Zelloidins nur die Stäbchen- oder die Schichtendoppelbrechung im Sinne O. Wi en er's in Betracht kommen. Besteht das System aus einer festen und einer flüssigen Komponente, so muB in beiden Fällen durch Veränderung des Brechungsvermögens der Flüssigkeit auch eine Veränderung in der Stärke der Gesamtdoppelbrechung eintreten. Mit Rücksicht auf die zweimalige Nullwirkung bei allmählicher Erhöhung des Brechungsexponenten $n_{2}$ waren zwei Möglichkeiten denkbar: entweder lag Stäbchendoppelbrechung vor, dann muBte die Doppelbrechung der anderen Komponente negativen Charakter besitzen; oder es handelte sich um Schichtendoppelbrechung, dann muBte die Doppelbrechung der anderen Komponente positiv sein. Bei den bleibend verlängerten Zelloidinstreifen kann, wie später noch ausführlicher zu begründen sein wird, als die veränderliche Komponente nur die Stäbchendoppelbrechung in Frage kommen; also muß die Doppelbrechung der anderen Komponente, d. h. in diesem Falle die der Stäbchen selbst, negativ sein. Hieraus ergeben sich zwei wichtige Folgerungen : erstens müssen die Teilchen, aus denen die Streifen aufgebaut sind, räumlich anisotrop sein, d. h. sie müssen eine der Stäbchenform sich nähernde Gestal! haben; und zweitens müssen diese Teilchen nicht bloß räumlich, sondern auch optisch anisotrop sein. Durch die starke bleibende Verlängerung werden die Stäbchen so orientiert, daf ihre Längsachsen einer Parallelstellung zustreben und somit ihre Gesamtwirkung optisch als eine von Null verschiedene Summe der Einzelwirkungen erscheint, während im nicht deformierten Zelloidin wegen der völlig regellosen Lagerung der Stäbchen diese Summe der Einzelwirkungen gleich Null werden muß, weil sie sich immer paarweise gegenseitig aufheben.

\footnotetext{
7) Zeitschr. f. Krystallogr. 52, 48-57 (1913).
}

Diese Folgerungen sind aber nur berechtigt, wenn der Verlauf der Gesamtdoppelbrechung und der aus ihr errechneten Stäbchendoppelbrechung mit den Forderungen übereinstimmt, die sich aus den Wiener'schen Gleichungen ergeben. Wie die Untersuchungen gezeigt haben, ist dies in der Tat in weitgehender Weise der Fall. Wenn auch die Kurve für die Stäbchendoppelbrechung nicht ganz mit derjenigen übereinstimmt, die für einen Idealfall gefunden wurde, so zeigt sie doch im wesentlichen ganz ähnliche Eigenschaften, wie aus den Darlegungen auf S. 280 in Mitteilung II hervorgeht. Besonders wichtig erscheint mir auch, daß ganz dieselben starken Verschiedenheiten in der Dispersion der Doppelbrechung auftreten, wie ein Vergleich der Tabellen VI und VIII sofort ergibt.

Ebenso wie das Verhalten des Zelloidins läßt auch das der Zellulose die gezogenen Folgerungen berechtigt erscheinen. Hier wie dort ist die gesetzmäßige Veränderung der Gesamtdoppelbrechung und der daraus zu bestimmenden Stäbchendoppelbrechung mit dem allmählichen Ansteigen der Werte von $n_{2}$ unverkennbar. Wenn auch der Verlauf der Kurven nicht ganz so gleichmäßig wie beim Zelloidin ist, indem an zwei Stellen geringe Störungen bemerkbar werden, so sind diese doch so geringfügig, daß sie die wesentlichen Uebereinstimmungen überhaupt nicht beeinträchtigen können. Das Auftreten der Minima an den Stellen, wo die Differenz $n_{1}-n_{2}=0$ wird, und das Bestehen der größten Gangunterschiede in den Flüssigkeiten, für die die Differenzen $n_{1}-n_{2}$ am höchsten sind, sowie die dazwischen liegenden stetigen Uebergänge lassen die große Aehnlichkeit in beiden Fällen mit genügender Sicherheit hervortreten; zumal jene geringen Störungen in anderer Weise eine ganz befriedigende Erklärung finden können. Auch daß in der Dispersion der Doppelbrechung dieselben starken Verschiedenheiten auftreten, wie sie sich theoretisch für ein solches Zwei-Stoff-System ergeben müssen, spricht entschieden für die Berechtigung jener Folgerungen.

Es könnte mir nun vielleicht eingewendet werden, dab die aus den Beobachtungen gezogenen Schlüsse gewissermaßen schon in den gemachten Voraussetzungen über die Mitwirkung von Stäbchendoppelbrechung versteckt seien. Solchen etwaigen Einwürfen möchte ich gleich jetzt in einigen Bemerkungen entgegentreten. Daß wir es in den untersuchten Körpern mit Systemen zu tun haben, die sich 
aus einer festen und einer flüssigen Komponente zusammensetzen, unterliegt jedenfalls keinem Zweifel. Es erscheint mir ferner als ganz sicher, dab die zur Imbibition verwendeten Flüssigkeiten weder beim Zelloidin noch bei der Zellulose irgend eine chemische Veränderung der festen Teilchen hervorrufen. Das Zelloidin verhält sich gegenüber dem Wasser und ebenso auch den verschieden konzentrierten Lösungen von Kaliumquecksilberjodid völlig indifferent, wie vielfache Vorversuche sowohl mit Zelloidinstreifen, wie auch mit unveränderten Nitrozellulosefasern von hohem Stickstoffgehalt bewiesen haben. Ebenso rufen weder Wasser, noch Alkohol, noch Benzylalkohol oder Monobromnaphthalin irgend eine chemische Aenderung der Zellulose hervor. Alle Flüssigkeiten, die eine Quellung oder eine merkbare Formveränderung an den Streifen bewirkt hatten, waren bei der Auswahl sorgfältig ausgeschieden worden. Auch daB die ganzen Aenderungen in der Gesamtdoppelbrechung beim Wechseln der Flüssigkeiten vollständig reversibel waren, spricht entschieden dafür, dab die chemischen und auch physikalischen Eigenschaften der festen Teilchen unverändert blieben. Es wäre ferner auch kaum zu erklären, warum beim Zelloidin nur bei einer ganz bestimmten Konzentration der Quecksilbersalzlösung das Minimum der Kurve erreicht wird, während sowohl bei einem nur wenig geringeren oder nur wenig höheren Wassergehalt der Lösung sogleich wieder ein Ansteigen des Gangunterschieds in demselben Sinne eintritt. Dasselbe gilt für das Verhalten der Zellulose; auch hier ist mit Sicherheit zu beobachten, wie nur bei einer ganz bestimmten Mischung das Minimum der Kurven erreicht wird. Ein nur geringer Zusatz von der höher oder der niedriger brechenden Flüssigkeit bewirkt sofort wieder ein Ansteigen des Gangunterschieds in dem gleichen Sinne. Alle diese Ueberlegungen führen, wie ich glaube, unbedingt zu dem Schlusse, dab es sich hier um eine mit dem Brechungsvermögen der flüssigen Komponente veränderliche Doppelbrechung handelt. Wären nun die festen Teilchen an sich optisch isotrop, so müfte bei Anwendung e in er Flüssigkeit, deren Brechungsexponent mit dem der Teilchen übereinstimmt, das System ebenfalls isotrop bleiben. Allerdings tritt beim Zelloidin, wie wir wissen, $z$ we i $m$ a 1 Isotropie auf, aber nur dann, wenn die Differenz $n_{1}-n_{2}$ nicht Null, sondern das eine Mal einen positiven und das andere $\mathrm{Mal}$ einen negativen Wert hat. Bei der Zellulose dagegen wird die Gesamtdoppelbrechung überhaupt bei keiner Flüssigkeit Null. Wird also $n_{1}-n_{2}=0$, so läßt sich bei beiden Körpern nicht Isotropie, sondern eine bestimmte Stärke der Doppelbrechung beobachten, die beim Zelloidin das negative, bei der Zellulose das positive Vorzeichen besitzt. Diese Doppelbrechung kann aber nur eine Eigenschaft der festen Komponente sein, und da die festen Teilchen durch die verschiedenen Flüssigkeiten keine Veränderungen erfahren, so muß sie bei allen Versuchen konstant bleiben. Ich habe sie deshalb von vornherein als Eigendoppelbrechung bezeichnet.

Noch auf eine weitere Beobachtung möchte ich kurz hinweisen, die zwar nichts Neues lehrt, die aber in bequemer Weise den entgegengesetzten Charakter der Eigendoppelbrechung in beiden Körpern bezeugt. Stellt man aus den stark gedehnten Streifen nicht zu dünne Querschnitte her, so kann man an diesen auch das Verhalten im sog. konvergenten Licht prüfen. Wählt man zunächst diejenigen Flüssigkeiten, bei denen $n_{1}-n_{2}=0$ ist, so erkennt man sofort, daß die Schnitte aus dem Zelloidin das Achsenbild eines negativen einachsigen und die aus Zellulose das eines positiven Kristalls zeigen. Beim Zelloidin kann man nun das negative Achsenbild leicht in ein positives umwandeln, wenn man z. B. die Schnitte in Wasser legt. Bei der Zellulose dagegen zeigen die Schnitte in allen Flüssigkeiten das positive Achsenbild. In den Zelloidinschnitten sieht man auch beim allmählichen Steigen des Wertes von $\mathrm{n}_{2}$ recht gut dieselben Erscheinungen, wie sie für die Achsenbilder verschiedener Varietäten von Ápophylliten seit langem bekannt sind.

\section{XI.}

In jedem System, das aus einer flüssigen und einer festen Komponente in stark dispersem Zustande besteht, kann nur dann optische Isotropie eintreten, wenn die Differenz $n_{1}-n_{2}$ verschwindet und die Teilchen völlig regellos gelagert sind. Dabei ist es gleichgiltig, ob die Teilchen selbst optisch isotrop oder anisotrop sind; denn auch im zweiten Falle müBte die Surnme der Einzelwirkungen Null sein. Bleibt $n_{1}-n_{2}=0$ und wird durch irgend einen Einflub die Lagerung der Teilchen in der Weise geändert, daß eine Ungleichwertigkeit der Richtungen in dem System zustande kommt, dann kann Doppelbrechung auftreten, wenn die Teilchen selbst anisotrop sind. Mag die Aenderung der Lagerung nun durch mechanische Defor- 
mation, durch ein elektrisches oder magnetisches Feld, oder auch durch irgend eine gerichtete Strömung erzielt werden; stets muB dann die Summe der Einzelwirkungen von Null verschieden sein. Das ist im wesentlichen auch der Sinn der in den letzten Jahren oft besprochenen Langevin'schen Orientierungshypothese, die besonders zur Erklärung der elektro- und magnetooptischen Doppelbrechung gewisser kolloider Lösungen aufgestellt worden ist. Auch die merkwürdigen optischen Eigenschaften der sog. flüssigen Kristalle, sowie manche Erscheinungen bei gerichteten Strömungen in gewissen kolloiden Lösungen sind auf eine Orientierung an sich schon optisch anisotroper Teilchen zurückgeführt worden ${ }^{8}$ ). Auf alle diese Dinge kann jedoch in dieser Mitteilung nicht näher eingegangen werden, es soll vielmehr nur darauf hingewiesen werden, daB derartige Anschauungen keineswegs neu sind. Schon vor mehr als 50 Jahren haben E. Brücke und C. Nägeli die Anisotropie der Fasern des Tier- und Pflanzenkörpers auf eine gleichsinnige, oder doch annähernd gleichsinnige Orientierung anisotroper Molekülkomp'exe zurückgeführt, und besonders $\mathrm{Nägeli}$ hat diese Anschauung in mehreren sehr exakten Arbeiten genauer zu begründen versucht; er nannte später diese Teilchen "Mizelle" und danach hat man seine Anschauung auch wohl als die Hypothese der kristallinischen Mizelle, oder kurz als Mizellarhypothese bezeichnet ${ }^{9}$ ). Sie behandelt keineswegs nur die optischen Eigenschaften der Zellhäute u. dergl., sondern auch die Quellbarkeit, die Wachstumserscheinungen, die Kohäsionsverhältnisse usw. Auch auf diese Dinge näher einzugehen konnte hier nicht meine Aufgabe sein; aber ich wollte doch auf jene Ansichten über den Aufbau der Zellhäute, besonders der Zellulosefasern hinweisen, weil ja die beiden Körper, die ich bei meinen Untersuchungen vorzugsweise benutzte, als Derivate der in der Natur vorkommenden Zellulose betrachtet werden müssen. Hält man an der $\mathrm{N}$ äge 1 l'schen Ansicht fest und berücksich-

8) Vgl. hierzu: W. Voigt, Elektrooptik und Magnetooptik (Hanđwörterbuch d. Naturw. 3, 483, u. 6, 712); ferner: Ders., Flüssige Kristalle und anisotrope Flüssigkeiten (Physik. Zeitschr. 17, 76-87, $128-135,152-161) ; H$. Diesselhorst und $H$. Freundlich, Ueber Schlierenbildung in kolloiden Lösungen und ein Verfahren, die Gestail der Kolloidteilchen festzustellen (Physik. Zeitschr. 17, 117--128, 1916).

9) Eine Zusammenstellung der Literatur hierüber findet sich in: V.v. E b n e r, Untersuchungen uber die Anisotropie organisierter Substanzen (Leipzig 1882). tigt man auBerdem die Vorgänge bei der Ent. stehung der Nitrozellulose, die fast vollkommene Erhaltung der Faserform und den dabei eintretenden Wechsel im Charakter der Doppelbrechung, so liegt die weitere Annahme nahe, daB die Form der Zellulosemizelle trotz dem starken chemischen Eingriff gar keine wesentliche Aenderung ihrer Gestalt erfährt. Auch bei der starken Auflockerung, die die Schießbaumwolle bei der Herstellung des Zelloidins erfährt, kann wohl nur eine völlige Desorientierung der Teilchen eintreten, ihre Einzelgestalt wird aber sicher beibehalten. Und wandelt man nun wieder das Zelloidin durch Denitrierung in Zellulose um, so wird, wie schon mehrfach angedeutet worden ist, auch hierbei keine irgendwie wesentliche Aenderung in der Form der Mizelle stattfinden. Wir kommen so auch auf einem ganz anderen Wege zu demselben Schluß, den wir bereits aus den Ergebnissen der Untersuchungen über den Verlauf . der Doppelbrechung in den bleibend verlängerten Streifen aus Zelloidin und Zellulose gezogen hatten, dab nämlich die festen Teilchen in diesen Systemen nicht nur räumlich, sondern auch optisch anisotrop seien. Die Teilchen haben eben stäbchenförmige Gestalt, und bei der starken bleibenden Deformation wird ihre frühere regellose Lagerung so verändert, daß sie nunmehr einer Orientierung zustreben, in der ihre Längsachsen mehr und mehr eine der Dehnungsrichtung sich nähernde Lage einnehmen. Aus dem optischen Verhalten der Nitrozellulosefasern, wie es sich aus den Untersuchungen Hans Ambron n's ${ }^{10}$ ) ergeben hat, dürfen wir ferner schlieben, daß die stäbchenförmige $\mathrm{Mi}$ zelle mit ihren Längsachsen parallel der Längsachse der Fasern gestellt ist; besonders die Eigenschaften der gefärbten Fasern machen dies sehr wahrscheinlich. Wie ich früher schon gezeigt habe, ergeben sich bei der Einlagerung von Farbstoffen in die verlängerten Zelloidinund Zellulosestreifen ganz dieselben Verschiedenheiten in der Absorption nach verschiedenen Richtungen. Es erscheint mir deshalb ganz berechtigt, wenn ich auch hieraus den Schluß ziehe, daB die festen Teilchen in diesen Körpern im wesentlichen dieselbe Gestalt haben, mögen sie nun in bezug auf ihre Längsachse positive oder negative Doppelbrechung besitzen.

Aus allen diesen auf verschiedenen Wegen gewonnenen Ergebnissen darf man nun wohl mit großer Sicherheit annehmen, dab zu der stets positiven Stäbchendoppelbrechung beim

io) Hans Ambronn, a. a. O. 30 flgde. 
Zelloidin negative und bei der Zellulose positive. Eigendoppelbrechung hinzukommt und daB das Zusammenwirken dieser beiden Komponenten den charakteristischen Verlauf der Gesamtdoppelbrechung, wie er sich bei meinen genauen Messungen darstellte, in ganz befriedigender Weise zu erklären vermag. Die Eigendoppelbrechung darf man wohl auch trotz den eingetretenen Spannungsdeformationen als konstant annehmen; denn es sind zum Erzeugen der bleibenden Verlängerungen nur schwache Spannungen nötig; das Zelloidin im imbibierten Zustande ist eine sehr plastische Masse, die schon durch einen schwachen Fingerdruck eine Veränderung der Form erfährt. Es ist deshalb kaum anzunehmen, da $B$ in einem solchen Körper, der nahezu 80 Proz. Flüssigkeit enthält, eine Deformation der Stäbchen selbst eintritt, es ist ohne $Z$ weifel viel wahrscheinlicher, daB nur eine Aenderung in ihrer Lage herbeigeführt wird. Wir haben es also mit einem ganz ähnlichen Vorgang zu tun, wie ich ihn früher bei einigen pflanzlichen Gummiarten angenommen habe und wie man ihn künstlich erzeugen kann, wenn man Fäden aus einer Mischung von Kolophonium und Wachs zieht. Auch auf diese Untersuchungen kann ich hier nicht näher eingehen, sondern muß auf die hierüber veröffentlichten Arbeiten verweisen ${ }^{11}$ ).

\section{XII.}

Die Werte für die Eigendoppelbrechung, wie sie aus den Gangunterschieden berechnet wurden, bei denen die Stäbchendoppelbrechung als ausgeschaltet betrachtet werden konnte, ergeben nun nicht etwa die Stärke der Doppelbrechung der Stäbchen selbst, aus denen die Systeme aufgebaut sind, sondern nur diejenige Differenz der Brechungsexponenten in zwei senkrecht aufeinander stehenden Richtungen, die aus der Summe der Einzelwirkungen entsteht. Erst wenn die Deformation soweit getrieben werden könnte, daß alle Stäbchen wirklich parallel orientiert wären, dann würde die Eigendoppelbrechung des Systems mit der der Einzelstäbchen übereinstimmen. Je stärker die bleibende Verlängerung ist, desto mehr muß die Richtung der Längsachsen der einzelnen Stäbchen von der regellosen Lage abweichen. Die Gesamtwirkung des Systems wird deshalb, zunächst ganz abgesehen von der hinzukommenden Stäb-

11) Ueber das optische Verhalten der Kutikula und der verkorkten Membranen (Ber. d. Deutsch. Bot. Ges. 6, 226-230, 1888), sowie die schon unter $\left.{ }^{2}\right)-4$ ) genannten Mitteilungen. chendoppelbrechung, von dem Grade der Deformation abhängen. Ein Streifen, der nur um 25 Proz. seiner ursprünglichen Länge gedehnt worden ist, zeigt natürlich eine geringere Doppelbrechung, als ein solcher, der eine bleibende Verlängerung um 100 Proz. erfahren hat. Um vergleichbare Ergebnisse bei den Messungen der Gangunterschiede zu erhalten, sind deshalb stets, wie schon anfangs erwähnt worden ist, in demselben Grade verlängerte Streifen verwendet worden; denn nur in diesem Falle darf man annehmen, daß die Stärke der Doppelbrechung der einen Komponente, also der Eigendoppelbrechung, konstant bleibt, wenn nach einander verschieden brechende Imbibilionsflüssigkeiten in das System eingeführt werden Aber nicht blob die Eigendoppelbrechung ist von dem Grade der Deformation abhängig sondern auch die Stäbchendoppelbrechung denn diese muß gleichfalls um so höher werden je mehr die Orientierung der Stäbchen sich der völligen Parallelstellung nähert. Sowohl die Eigendoppelbrechung wie die Stäbchendoppelbrechung muß deshalb stets niedriger sein, als in einem System, in dem eine vollkommen parallele Anordnung der Stäbchen vortıanden ist. Da dieser Grenzfall aber durch mechanische Deformation wohl kaum erreicht werden kann, so wird sich für jeden Grad der Veılängerung auch eine bestimmte Stärke der Eigendoppelbrechung und für den Wert von $\mathrm{n}_{2}$ eine bestimmte Stärke der Stäbchendoppelbrechung ergeben. Besitzen beide entgegengesetztes Vorzeichen, so muß die Gesamtdoppelbrechung dann Null werden, wenn die aus der Stärke der beiden Anisotropien sich ergebenden Gangunterschiede absolut gleich werden. Daß dieser Fall bei demselben Wert von $\mathrm{n}_{2}$ für verschiedene Grade der Verlängerung eintritt, ist von vornherein unwahrscheinlich; denn die Veränderung der Eigendoppelbrechung und die der Stäbchendoppelbrechung bei zunehmender Deformation stehen wohl sicher nicht in so einfacher Beziehung zu einander. Es muß also, wie übrigens die bereits angestellten Versuche bestätigt haben, der Nullwert für den Gangunterschied des ganzen Systems bei Verschiedenheit im Grad der Deformation auch bei verschiedenen Werten von $n_{2}$ auftreten.

Es ist nun von besonderem Interesse, dieses Wandern der Nullwerte durch genaue Messungen für verschiedene Wellenlängen zu verfolgen. Man kann dabei entweder in der Weise verfahren, daß man an demselben Streifen, der mit einer Flüssigkeit von bestimmtem Brech- 
ungsexponenten durchtränkt ist, bei fortschreitender Dehnung beobachtet, oder indem man Streifen von bestimmter Verlängerung nacheinander mit verschiedenen Flüssigkeiten imbibiert und dann feststellt, bei welchen Werten von $n_{2}$ der Gangunterschied auf Null herabgeht. Auf diese Weise den EinfluB des Deformationsgrades auf die Stärke der Gesamtdoppelbrechung zu prüfen, ist natürljech nuur beim Zelloidin möglich; denn nur hier kann eine solche Nullwirkung erwartet werden. Aber auch bei der Zellulose kann man die Unterschiede untersuchen, die sich bei verschiedener Stärke der Deformation geltend machen. Wie die bisher angestellten Versuche ergeben haben, wird mit zunehmender Deformation sowohl die Stärke der Stäbchendoppelbrechung wie die der Eigendoppelbrechung gesteigert. Die genaueren Messungen dieser Veränderungen sind noch keineswegs abgeschlossen, aber es darf schon jetzt hervorgehoben werden, daß die Beziehungen zwischen dem Deformationsgrad und der Gesamtdoppelbrechung sowohl wie der Eigenund Stäbchendoppelbrechung im besonderen manchen interessanten Aufschlub zu geben versprechen. Hat man einmal die genauen Zahlen für die Stärke der Doppelbrechung bei 25, $50,75,100$ Proz. bleibender Verlängerung, so kann man daraus vielleicht auch durch Extrapolation die Werte für noch beträchtlich stärkere Deformationen wenigstens annäherungsweise erhalten, und aus diesen werden sich dann eher Schlüsse auf das Verhalten bei vollständiger Parallelstellung der Stäbchen ziehen lassen. Hierüber hoffe ich in einer weiteren Mitteilung eingehender berichten zu können.

Eine viel größere Annäherung an völlig parallele Orientierung der Stäbchen oder Mizelle ist jedenfalls in vielen Zellhäuten von Pflanzen verwirklicht, besonders in den sehr langgestreckten Bastfasern, wie sie manche Nesselarten besitzen. Solche Zellen sind oft über $20 \mathrm{~cm}$ lang, und ihre Wände zeigen eine Stärke der Doppelbrechung, die die des Quarzes mehr als sechsmal übertrifft. Hieraus könnte man eher auf die Stärke der Doppelbrechung der Zellulosestäbchen schlieben; denn jene Membranen verhalten sich innerhalb kleiner Flächengebiete fast wie einheitliche Kristalle. Ihr gesamter Aufbau stellt allerdings wegen der radialen und konzentrischen Anordnung der optischen Symmetrieachsen ein zylinderförmiges Kristallaggregat dar. Vergleicht man nun mit der Doppelbrechung dieser Zellulose die aus den vorstehenden Messungen ermittelte, so fällt der große Unterschied auf; denn das System der Stäbchen in den bleibend verlängerten Zellulosestreifen besitzt bei mittlerer Wellenlänge für die Differenz $n_{a}-n_{0}$ nur den Wert 0,013 , die Stärke der Doppelbrechung ist also nur wenig gröBer als die des Quarzes, die 0,009 für dieselbe Wellenlänge beträgt. Diese große Verschiedenheit erscheint wohl etwas überraschend, sie erklärt sich aber leicht daraus, daB in den untersuchten Zellulosestreifen der Idealfall der völligen Parallelstellung der Stäbchen sicher bei weitem noch nicht erreicht ist. Außerdem ist wohl auch anzunehmen, daB die Doppelbrechung der durch Denitrierung gewonnenen Zellulosemizelle etwas geringer ist, als die der Zellwände in den Nesselfasern. Auch für die Nitrozellulose besteht ein ähnlicher Unterschied. Wie aus früheren Beobachtungen ${ }^{12}$ ) hervorgeht, besitzen die nitrierten Ramiefasern von 13,16 Proz. Stickstoffgehalt für die Wellenlänge $546 \mu \mu$ einen Gangunterschied von 0,32 ; daraus berechnet sich bei einer Dicke von $20 \mu$ die Stärke der Doppelbrechung $n_{0}-n_{a}=0,008$. $\mathrm{Nach}$ den angenäherten Bestimmungen der entsprechenden Zahl für die gedehnten Zelloidinstreifen ergibt sich dagegen für dieselbe Wellenlänge nur 0,001. Erst wenn man in der Lage wäre, nach der vorhin angedeuteten $\mathrm{Me}$ thode die Stärke der Doppelbrechung für völlige Parallelstellung der Stäbchen zu ermitteln, könnte man auch einen genaueren quantitativen Vergleich dieser Zahlen anstellen.

Am Schlusse dieser Mitteilung möchte ich nochmals auf diejenigen Ergebnisse hinweisen, denen meines Erachtens eine allgemeinere Bedeutung zukommt. Die Messungen haben bewiesen, daß die Doppelbrechung der untersuchten Körper in einer bestimmten Weise von dem Brechungsvermögen der eingelagerten Flüssigkeit abhängt. Die Kurve der Gangunterschiede zeigt in beiden Fällen ein Minimum, wenn das Brechungsvermögen der festen Teilchen und das der Flüssigkeit übereinstimmt. Es wurde daraus der Schluß gezogen, daß die beobachtete Doppelbrechung durch das Zusammenwirken zweier Komponenten zustande kommt. Davon bleibt die eine bei der Einlagerung verschieden brechender Flüssigkeiten konstant; sie muB als eine Eigenschaft des Systems der festen Teilchen betrachtet werden, und ich habe sie deshalb als Eigendoppelbrechung bezeichnet. - Die andere dagegen ist mit dem Brechungsvermögen der Flüssigkeit veränderlich, und auf

12) Hans Ambronn a. a. O. 24. 
Grund verschiedener Ueberlegungen kam ich zu dem weiteren Schluß, dab diese zweite Komponente als Stäbchendoppelbrechung im Sinne $O$. Wi en er's aufzufassen sei. Ist aber diese Folgerung berechtigt, dann müssen die festen Teilchen in den untersuchten Systemen stäbchenförmige Gestalt besitzen und durch die bleibende Verlängerung so orientiert werden, daf ihre Längsachsen einer Parallelstellung mit der Dehnungsrichtung zustreben. Je stärker die Verlängerung ist, desto mehr wird die Anordnung der vorher regellos gelagerten Teilchen sich diesem Zustand nähern, und desto stärker muß sowohl die Eigendoppelbrechung wie die Stäbchendoppelbrechung des Systems werden. Besitzt die Eigendoppelbrechung, wie dies beim Zelloidin der Fall ist, das negative Vorzeichen, so muß in zwei bestimmten Fällen als Resultierende optische Isotropie eintreten, was die Versuche bestätigt haben. Gerade dieser Umstand scheint mir besondere Beweiskraft für die vorgetragene Ansicht zu haben.

Es wäre sehr erwünscht, wenn noch auf anderem Wege die räumliche Anisotropie der Teilchen und der Uebergang aus der regellosen Lagerung in eine mehr orientierte Anordnung bewiesen werden könnte. Es liegt nahe, an die Durchstrahlung der Streifen mit Röntgenstrahlen zu denken. Jedoch glaube ich, dab dabei kein befriedigendes Ergebnis zu erhalten wäre, weil die gleichsinnige Orientierung selbst bei sehr starker Verlängerung keineswegs so weit vorgeschritten ist, dab sich wie bei einem einheitlichen Kristall ein charakteristisches $\mathrm{L}$ a u eDiagramm ergeben würde. Auch F. R in n e ${ }^{13}$ ) hat an stark gedehnten Zelluloidstreifen keine Wirkung in diesem Sinne erhalten. Mehr Aussicht auf Erfolg dürften dagegen Versuche haben, die nach der Methode von P. Debye und P.

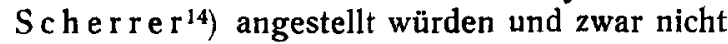

19) F. Rinne, Beiträge zur Kenntnis der KristallRöntgenogramme (Ber. der Kgl. Sächs. Ges. der Wiss. Math.- phys. Kl. 67, 312, 1915 ).

14) P. Debye und P. Scherrer, Interferenzen aus regellos orientierten Teilchen im Röntgenlicht, I an den gedehnten, sondern an den noch isotropen Streifen. Nach den theoretischen Darlegungen und den Versuchen dieser beiden Forscher kann man bei Durchstrahlung sog. amorpher Körper mit Röntgenstrahlen aus den Interferenzerscheinungen feststellen, ob ein kristallinischer oder amorpher Zustand vorliegt. Die Untersuchungen wurden mit feinen Pulvern verschiedener Körper, wie amorphem Bor, amorphem Silizium, Lithiumfluorid u. a. angestellt, und ergaben mit Sicherheit den kristallinischen Zustand dieser Körper. Debye und $S$ cherrer weisen auch darauf hin, daB die Interferenzen, die W Friedrich ${ }^{15}$ ) bei der Durchstrahlung von Wachs und Paraffin erhalten hatte, für das Vorhandensein regellos gelagerter sehr kleiner Kriställchen in diesen Körpern sprechen. Daß eine solche mikrokristallinische Struktur bei Wachs und Paraffin sowie bei vielen Fetten u. dergl. in der Tat besteht, darauf haben schon Versuche von $D$. Brewster aus der Mitte des vorigen Jahrhunderts schließen lassen ${ }^{16}$ ). Man kann übrigens die zahllosen sehr kleinen Kriställchen $z$. B. in einer dünnen Wachsschicht im Polarisationsmikroskop bei stärkerer VergröBerung sofort erkennen. Würde man nun bei der Durchstrahlung frischer Zelloidinstücke ähnliche Interferenzwirkungen erhalten, so wäre damit ein sehr erwünschter weiterer Beweis für die räumliche und optische Anisotropie der darin enthaltenen festen Teilchen erbracht. Leider bin ich zur Zeit nicht in der Lage, derartige Versuche anzustellen.

(Nachr. d. Kgl. Ges. d. Wiss. in Gottingen Math.-phys. KI. 47, 1-15, 1916, und Physik. Zeitschr. 17, 277 bis $283,1916)$. (1913)

15) W. Friedrich, Physik. Zeitschr. 14, 317

16) D. Brewster, On the production of crystalline structure in crystallised powders by compression and traction (Trans. of the Roy. Soc. of Edinburgh 20. 558,1853 ). Vgl. auch die oben schon unter $\left.{ }^{2}\right)$, 4) and 11) zitierten Arbeiten, sowie S. S chwendener, Gesammelte botan. Abhandl. 1, 375 (1898). 